\title{
Weak and strong regimes of incompressible magnetohydrodynamic turbulence
}

\author{
G. Gogoberidze, ${ }^{1,2}$ S. M. Mahajan, ${ }^{3}$ and S. Poedts ${ }^{1}$ \\ ${ }^{1}$ Centre for Plasma Astrophysics, K.U.Leuven, \\ Celestijnenlaan 200B, 3001 Leuven, Belgium. \\ ${ }^{2}$ Georgian National Astrophysical Observatory, \\ 2 a Kazbegi ave., 0160 Tbilisi, Georgia. \\ ${ }^{3}$ Institute for Fusion Studies, University of Texas at Austin, Austin, TX 78712.
}

(Dated: February 20, 2013)

\begin{abstract}
It is shown that in the framework of the weak turbulence theory, the autocorrelation and cascade timescales are always of the same order of magnitude. This means that, contrary to the general belief, any model of turbulence which implies a large number of collisions among wave packets for an efficient energy cascade (such as the Iroshnikov-Kraichnan model) are not compatible with the weak turbulence theory.

PACS numbers: 52.35.Ra, 47.27.Gs, 47.27.Eq
\end{abstract}




\section{INTRODUCTION}

Magnetohydrodynamic (MHD) turbulence is present in a wide variety of astrophysical systems such as the solar wind, the interstellar medium, accretion discs, and so on. Incompressible MHD is the standard model for the study of astrophysical MHD turbulence. Although incompressible MHD turbulence has been intensively studied for the last several decades [1, 2, 3, 4, 5, 6, 7, 8, 9, 10, 11, 12, 13, 14, 15, 16, 17, 18] many physical aspects of the problem still remain unclear. The first model of incompressible MHD turbulence was proposed by Iroshnikov [1] and Kraichnan [2]. The Iroshnikov-Kraichnan (IK) model of MHD turbulence is based on the fact that nonlinear interaction is possible only among Alfvén waves propagating in opposite directions along the mean magnetic field. Therefore, an energy cascade occurs as a result of collisions between oppositely propagating Alfvén waves. Consider the isotropic excitation of Alfvén waves on some outer scale $l_{0}$ with a characteristic velocity $v_{0} \ll V_{A}$, where $V_{A}$ denotes the Alfvén velocity. The IK model assumes that the energy transfer is local and isotropic in the wave number space. The characteristic time scale of the Alfvén wave collision is $\tau_{a c}^{I K} \sim\left(k V_{A}\right)^{-1}$, where $k$ is the wave number. Using the governing equations of incompressible MHD, it can be shown that during one collision the distortion of each wave packet $\delta v_{l}$ is of the order $\delta v_{l} / v_{l} \sim v_{l} / V_{A} \ll 1$. Because these perturbations are summed with random phases $N \sim\left(v_{l} / \delta v_{l}\right)^{2} \sim\left(V_{A} / v_{l}\right)^{2}$, collisions are necessary to achieve the distortion of order unity. Therefore, for the energy cascade time $\tau_{\text {cas }}^{I K}$ we have

$$
\tau_{\text {cas }}^{I K} \sim \frac{1}{k v_{l}} \frac{V_{A}}{v_{l}}
$$

Taking into account the relations $\varepsilon \sim v_{l}^{2} / \tau_{c a s}$ and $v_{l}^{2} \sim k \mathcal{E}_{k}$, where $\varepsilon$ is the energy cascade rate and $E_{k}$ is the one dimensional energy spectrum, we obtain

$$
\mathcal{E}_{k}^{I K} \sim\left(\varepsilon V_{A}\right)^{1 / 2} k^{-3 / 2}
$$

which represents the IK spectrum of incompressible MHD turbulence. Due to the condition $v_{0} \ll V_{A}$ the IK model is usually deemed as the model of weak MHD turbulence (i.e., the model for which perturbation theory called weak turbulence theory (WTT) [19, 20] is applicable).

The IK model for MHD turbulence is isotropic. However, the presence of a mean magnetic field has a strong effect on the turbulence properties, in contrast to a mean flow in 
hydrodynamic turbulence, which can be eliminated by the corresponding Galilean transformation. The anisotropy of MHD turbulence had been seen in various numerical simulations [3, 8, 10, 12]. Different theoretical models of weak [5, 7, 9] as well as strong [4, 6, 17, 18] anisotropic MHD turbulence have been developed to the date. In Ref. [3] it was shown that the lowest order three wave interactions among Alfvén waves are possible only if the parallel (with respect to the constant magnetic field) wave number of one mode is zero. This implies that in the framework of WTT there is no parallel cascade of energy. Consequently, the turbulence is anisotropic and the energy is cascaded to larger values of the perpendicular wave number.

Comprehensive study of the MHD turbulence in the framework of the WTT has been performed in Ref. [9], where the full coupled equation for shear and pseudo Alfvén waves has been derived and analyzed. Stationary solutions of the WTT equations has been found and was shown that for balanced turbulence the three dimensional energy spectrum $E\left(k_{\|}, k_{\perp}\right) \sim$ $\varepsilon^{1 / 2} k_{\perp}^{-3}$. The validity criterium of the WTT for anisotropic MHD turbulence is [6]

$$
\frac{k_{\perp} v_{l}}{k_{\|} V_{A}} \ll 1
$$

The usual interpretation of this condition implies that the nonlinear strain time should be less then the wave period.

In the present paper we study incompressible MHD turbulence in the framework of the weak coupling approximation (WCA) [19], which represents one of the equivalent formulations of the direct interaction approximation (DIA) 22]. The WCA allows us to study both weak and strong limits of MHD turbulence in the framework of a unified formalism. In the case of zero residual energy we rederive the WTT equations from the WCA equations of incompressible MHD turbulence derived earlier in Ref. [9]. We show that in the framework of the WTT the autocorrelation and cascade (evolution) timescales are always of the same order of magnitude. This means that, contrary to the general belief, in the WTT $N \sim 1$ and, consequently, any model for MHD turbulence that implies $N \gg 1$ (such as the IK model) are incompatible with the WTT.

The paper is organized as follows. The WCA formalism for incompressible MHD turbulence is described in Sec. III. The WTT equations are derived and analyzed in Sec. III. The conclusions are given in Sec. IV] 


\section{THE WCA EQUATIONS FOR INCOMPRESSIBLE MHD TURBULENCE}

Consider incompressible MHD turbulence in the presence of a constant magnetic field $\mathbf{B}_{0}$ directed along $z$ axis. The equations of ideal MHD, governing the evolution of fluctuations of the Elsasser variables, are

$$
\begin{gathered}
\partial_{t} \mathbf{U}_{1}-\mathbf{V}_{A} \partial_{z} \mathbf{U}_{1}=-\left(\mathbf{W}_{1} \cdot \nabla\right) \mathbf{U}_{1}-\nabla p \\
\partial_{t} \mathbf{W}_{1}+\mathbf{V}_{A} \partial_{z} \mathbf{W}_{1}=-\left(\mathbf{U}_{1} \cdot \nabla\right) \mathbf{W}_{1}-\nabla p
\end{gathered}
$$

where $\mathbf{V}_{A} \equiv \mathbf{B}_{\mathbf{0}} / \sqrt{4 \pi \rho}$ is the Alfvén velocity, $\mathbf{W}_{1}=\mathbf{v}_{1}+\mathbf{b}_{1}$ and $\mathbf{U}_{1}=\mathbf{v}_{1}-\mathbf{b}_{1}$ are fluctuations of the Elsasser variables, $\mathbf{v}_{1}$ is the turbulent velocity field, $\mathbf{b}_{1} \equiv \mathbf{B}_{1} / \sqrt{4 \pi \rho}$ denotes the magnetic field of the fluctuations in velocity units, $p$ is the pressure normalized by the density and $\partial_{t} \equiv \partial / \partial t$.

Performing a Fourier transform, neglecting pseudo Alfvén waves which are known to play only a passive role for strongly anisotropic MHD turbulence [6], defining the unit polarization vector of the shear Alfvén waves as $\hat{\mathbf{e}}_{\mathbf{k}}=\hat{\mathbf{k}} \times \mathbf{z}$, and introducing the amplitudes of the shear Alfvén waves as

$$
\mathbf{w}_{\overline{\mathbf{k}}}=i \phi_{\overline{\mathbf{k}}} \hat{\mathbf{e}}_{\mathbf{k}}, \quad \mathbf{u}_{\overline{\mathbf{k}}}=i \psi_{\overline{\mathbf{k}}} \hat{\mathbf{e}}_{\mathbf{k}}
$$

Eqs. (4)-(5) reduce to the following set of equations

$$
\begin{aligned}
& \left(\omega-\omega_{\mathbf{k}}\right) \phi_{\overline{\mathbf{k}}}=\int_{-\infty}^{\infty} T_{1,2} \phi_{1} \psi_{2} d \mathcal{F}_{1,2}^{k}, \\
& \left(\omega+\omega_{\mathbf{k}}\right) \psi_{\overline{\mathbf{k}}}=\int_{-\infty}^{\infty} T_{1,2} \psi_{1} \phi_{2} d \mathcal{F}_{1,2}^{k},
\end{aligned}
$$

where $T_{1,2} \equiv i\left(\hat{\mathbf{e}}_{\mathbf{k}} \cdot \hat{\mathbf{e}}_{\mathbf{k}_{\mathbf{1}}}\right)\left(\mathbf{k} \cdot \hat{\mathbf{e}}_{\mathbf{k}_{\mathbf{2}}}\right)$ is the matrix element of interaction.

Applying the standard technique of WCA, one can then obtain the following set of equations [17]

$$
\begin{gathered}
\zeta_{\overline{\mathbf{k}}}^{+}=\int_{-\infty}^{\infty}\left|T_{1,2}\right|^{2} \Gamma_{1}^{+} I_{2}^{-} d \mathcal{F}_{1,2}^{k}, \\
-i\left(\omega-\omega_{\mathbf{k}}\right) I_{\overline{\mathbf{k}}}^{+}=\Gamma_{\overline{\mathbf{k}}}^{+*} \int_{-\infty}^{\infty}\left|T_{1,2}\right|^{2} I_{1}^{+} I_{2}^{-} d \mathcal{F}_{1,2}^{k}- \\
I_{\overline{\mathbf{k}}}^{+} \int_{-\infty}^{\infty}\left|T_{1,2}\right|^{2} \Gamma_{1}^{+} I_{2}^{-} d \mathcal{F}_{1,2}^{k},
\end{gathered}
$$

and similar equations for $\zeta_{\overline{\mathbf{k}}}^{-}$and $I_{\overline{\mathbf{k}}}^{-}$. Here $\overline{\mathbf{k}} \equiv(\mathbf{k}, \omega)$, the caret denotes the unit vector, $\mathbf{u}_{1}$ denotes $\mathbf{u}_{\overline{\mathbf{k}}_{1}}, \omega_{\mathbf{k}}=k_{z} V_{A}$ is the frequency of the Alfvén wave, $d \mathcal{F}_{1,2}^{k} \equiv d^{4} \overline{\mathbf{k}}_{1} d^{4} \overline{\mathbf{k}}_{2} \delta_{\overline{\mathbf{k}}-\overline{\mathbf{k}}_{1}-\overline{\mathbf{k}}_{2}}$, and 
$\delta_{\overline{\mathbf{k}}-\overline{\mathbf{k}}_{1}-\overline{\mathbf{k}}_{2}} \equiv \delta\left(\overline{\mathbf{k}}-\overline{\mathbf{k}}_{1}-\overline{\mathbf{k}}_{2}\right)$ is the Dirac delta function,

$$
\left\langle\phi_{\overline{\mathbf{k}}} \phi_{\overline{\mathbf{k}}^{\prime}}^{*}\right\rangle=I_{\overline{\mathbf{k}}}^{+} \delta_{\overline{\mathbf{k}}-\overline{\mathbf{k}}^{\prime}}, \quad\left\langle\psi_{\overline{\mathbf{k}}} \psi_{\overline{\mathbf{k}}^{\prime}}^{*}\right\rangle=I_{\overline{\mathbf{k}}}^{-} \delta_{\overline{\mathbf{k}}-\overline{\mathbf{k}}^{\prime}},
$$

and

$$
\Gamma_{\overline{\mathbf{k}}}^{ \pm}=\frac{i}{\omega \mp \omega_{\mathbf{k}}+i \zeta_{\overline{\mathbf{k}}}^{ \pm}}
$$

Eqs. (9)-(10) are useless unless some assumptions are made about the frequency dependence of $\Gamma_{\overline{\mathbf{k}}}^{ \pm}$and $I_{\overline{\mathbf{k}}}^{ \pm}$. Equivalently, in the framework of the DIA one should make some assumptions about the time dependence of $G^{ \pm}(\mathbf{k}, \tau)$ and $Q^{ \pm}(\mathbf{k}, \tau)$ [21], which are the corresponding inverse Fourier transforms of $\Gamma_{\overline{\mathbf{k}}}^{ \pm} / 2 \pi$ and $I_{\overline{\mathbf{k}}}^{ \pm}$with respect to $\omega$ [i.e., $\left.\left\langle\phi_{\mathbf{k}}(t+\tau) \phi_{\mathbf{k}^{\prime}}^{*}(t)\right\rangle=Q^{+}(\mathbf{k}, \tau) \delta_{\mathbf{k}-\mathbf{k}^{\prime}}\right]$. One of the simplest and frequently used assumptions implies [21, 22]

$$
\begin{gathered}
G^{ \pm}(\mathbf{k}, \tau)=\exp \left(-\left|\eta_{\mathbf{k}}^{ \pm}\right| \tau \pm i \omega_{\mathbf{k}} \tau\right) H(\tau), \\
Q^{ \pm}(\mathbf{k}, \tau)=\exp \left(-\left|\eta_{\mathbf{k}}^{ \pm}\right| \tau \pm i \omega_{\mathbf{k}} \tau\right) E_{\mathbf{k}}
\end{gathered}
$$

where $H(t)$ is the Heaviside (step) function, and $E_{\mathbf{k}}$ is the energy spectrum.

Similar to Eqs. (13)-(14), in the case under consideration we assume

$$
\begin{gathered}
\zeta_{\overline{\mathbf{k}}}^{ \pm}=\eta_{\mathbf{k}}^{ \pm}, \\
I_{\overline{\mathbf{k}}}^{ \pm}=\frac{E_{\mathbf{k}}^{ \pm}}{\pi} \frac{\eta_{\mathbf{k}}^{ \pm}}{\left(\omega \mp \omega_{\mathbf{k}}\right)^{2}+\left(\eta_{\mathbf{k}}^{ \pm}\right)^{2}} .
\end{gathered}
$$

Here, for simplicity, we consider the symmetric case $\eta_{\mathbf{k}}^{ \pm}=\eta_{\mathbf{k}}$, and $E_{\mathbf{k}}^{ \pm}=E_{\mathbf{k}}$, which physically corresponds to a turbulence with zero cross helicity. Although we consider the symmetric case, in the further analysis we will keep the \pm signs for the energy spectra in order to underline the fact that nonlinear interactions are possible only between counter propagating modes.

$\tau_{a c} \equiv 1 / \eta_{\mathbf{k}}$ is the autocorrelation time. As it was mentioned in Refs. [2, 19] the random Galilean invariance requires that before applying the WCA (DIA) closure scheme to Eqs. (77)(8) one should remove the influence of the velocity field of low frequency modes (for a more detailed analysis see Ref. [17]). If this is done, and the corresponding contributions are removed from Eqs. (9)-(10), then $\tau_{a c}$ represents the Lagrangian autocorrelation timescale, which is called "the duration of unit act of interaction" in heuristic models of the turbulence. 


\section{DERIVATION AND ANALYSIS OF THE WTT EQUATIONS}

Substituting Eqs. (15) and (16) into Eq. (9) and performing an integration with respect to the frequencies, we get

$$
\begin{gathered}
-i\left(\omega-\omega_{\mathbf{k}}\right) I_{\overline{\mathbf{k}}}^{+}=\Gamma_{\overline{\mathbf{k}}}^{+*} \int_{-\infty}^{\infty}\left|T_{1,2}\right|^{2} R_{a} E_{1}^{+} E_{2}^{-} \mathrm{d} \mathcal{K}_{1,2}^{k}- \\
I_{\overline{\mathbf{k}}}^{+} \int_{-\infty}^{\infty}\left|T_{1,2}\right|^{2} R_{b} E_{2}^{-} \mathrm{d} \mathcal{K}_{1,2}^{k}, \\
\eta_{\mathbf{k}}=\int_{-\infty}^{\infty}\left|T_{1,2}\right|^{2} R_{b} E_{2}^{-} \mathrm{d} \mathcal{K}_{1,2}^{k},
\end{gathered}
$$

where

$$
\begin{gathered}
R_{a}=\frac{1}{\pi} \frac{\eta_{1}+\eta_{2}}{\left(\eta_{1}+\eta_{2}\right)^{2}+\left(\omega-\omega_{k}+\Delta \omega_{k 12}\right)^{2}} \\
R_{b}=\frac{1}{\left(\eta_{1}+\eta_{2}\right)-i\left(\omega-\omega_{k}+\Delta \omega_{k 12}\right)},
\end{gathered}
$$

$\mathrm{d} \mathcal{K}_{1,2}^{k} \equiv \mathrm{d}^{3} \mathbf{k}_{1} \mathrm{~d}^{3} \mathbf{k}_{2} \delta_{\mathbf{k}-\mathbf{k}_{1}-\mathbf{k}_{2}}$, and $\Delta \omega_{k 12}=\omega_{\mathbf{k}}-\omega_{\mathbf{k}_{1}}+\omega_{\mathbf{k}_{2}}$.

According to Eq. (16) $), I_{\overline{\mathbf{k}}}^{ \pm}$significantly differs from zero when $\left(\omega-\omega_{\mathbf{k}}\right) \lesssim \eta_{\mathbf{k}}$. First of all, consider the real part of the right hand side of Eq. (17) which describes the nonlinear decay of the fluctuations (whereas the real part describes the frequency shift caused by nonlinear interactions). Consider the limit $\eta_{\mathbf{k}} \rightarrow 0$. In this case, the width of the wave packets tends to zero and Eq. (16) yields $I_{\overline{\mathbf{k}}}^{ \pm}=E_{\mathbf{k}}^{ \pm} \delta_{\omega \mp \omega_{\mathbf{k}}}$. In the considered limit the integrals on the right hand side of Eq. (17) are dominated by the contribution of the small vicinity of the so-called resonant curve (defined by the condition $\Delta \omega_{k 12}=0$ ) where the condition

$$
\Delta \omega_{k 12} \lesssim \eta_{\mathbf{k}}
$$

is fulfilled. The solution of the resonant condition $\Delta \omega_{k 12}=0$ is $k_{z 2}=0$. Consequently, three wave resonant interactions must include the zero frequency mode [3, 7, 9]. The volume of the wave number space occupied by the resonant area where the condition (21) is fulfilled is $\left[\eta_{\mathbf{k}} /\left(\partial \omega_{k} / \partial k_{z}\right)\right] k_{\perp}^{2}$. Taking also into account that a typical value of $R_{a}$ and $\Re\left(R_{b}\right) / \pi$ in the resonant area is $1 / \eta_{\mathbf{k}}$, noting that $T_{1,2} \sim k_{\perp}$ and assuming that the nonlinear energy transfer in the $\mathbf{k}_{\perp}$ plane is dominated by triad interactions with $k_{\perp} \sim k_{\perp 1,2}$ then the contribution of the resonant area in (say) the first integral of Eq. (17) can be estimated as $k_{\perp}^{4} E^{+}\left(k_{z}, k_{\perp}\right) E^{-}\left(0, k_{\perp}\right) /\left(\partial \omega_{k} / \partial k_{z}\right)$. Similarly, the contribution of the rest part of the 
$\mathbf{k}$ space is $\eta_{\mathbf{k}} k_{z} k_{\perp}^{4} E^{+}\left(k_{z}, k_{\perp}\right) E^{-}\left(k_{z}, k_{\perp}\right) / \omega_{k}^{2}$. Consequently, the domination of the resonant contribution implies

$$
\frac{\eta_{\mathbf{k}}}{\omega_{\mathbf{k}}} \frac{E^{-}\left(k_{z}, k_{\perp}\right)}{E^{-}\left(0, k_{\perp}\right)} \ll 1
$$

If this condition is fulfilled, one can replace $R_{a}$ and $\Re\left(R_{b}\right) / \pi$ by $\delta\left(\omega_{\mathbf{k}}-\omega_{\mathbf{k}_{1}}+\omega_{\mathbf{k}_{2}}\right) \equiv \delta_{\Delta \omega_{k 12}}$. Then, the real part of Eq. (17) reduces to

$$
-\gamma_{\mathbf{k}} E_{\mathbf{k}}^{+}=\pi \int_{-\infty}^{\infty}\left|T_{1,2}\right|^{2} E_{2}^{-}\left(E_{1}^{+}-E_{\mathbf{k}}^{+}\right) \delta_{\Delta \omega_{k 12}} \mathrm{~d} \mathcal{K}_{1,2}^{k},
$$

where $\gamma_{\mathbf{k}}$ is the total decrement caused by the nonlinear interactions.

In contrast to the DIA which operates with a two point two time correlation functions and/or their Fourier transforms $\left(Q^{ \pm}(\mathbf{k}, \tau)\right.$ and $\left.I_{\overline{\mathbf{k}}}^{ \pm}\right)$, the WTT implies Markovian closure and consequently operates with the Fourier transform of a two point one time correlation function $n_{\mathbf{k}}^{ \pm}(t)$ defined as $\left\langle\phi_{\mathbf{k}}(t) \phi_{\mathbf{k}^{\prime}}^{*}(t)\right\rangle=n_{\mathbf{k}}^{ \pm}(t) \delta_{\mathbf{k}-\mathbf{k}^{\prime}}$. As known, the nonlinear decrement of a one time correlation function for a zero time separation is twice larger then the nonlinear decrement of a two point correlation function since all temporal derivatives in the dynamic equations now act on both the time variables [21]. Consequently, according to Eq. (223) the dynamic equation for $n_{\mathbf{k}}^{+}(t)$ is

$$
\frac{\partial n_{\mathbf{k}}^{+}}{\partial t}=2 \pi \int_{-\infty}^{\infty}\left|T_{1,2}\right|^{2} n_{2}^{-}\left(n_{1}^{+}-n_{\mathbf{k}}^{+}\right) \delta_{\Delta \omega_{k 12}} \mathrm{~d} \mathcal{K}_{1,2}^{k} .
$$

Similar manipulations lead to the following equation for $n_{\mathbf{k}}^{-}(t)$

$$
\frac{\partial n_{\mathbf{k}}^{-}}{\partial t}=2 \pi \int_{-\infty}^{\infty}\left|T_{1,2}\right|^{2} n_{2}^{+}\left(n_{1}^{-}-n_{\mathbf{k}}^{-}\right) \delta_{\Delta \omega_{k 12}} \mathrm{~d} \mathcal{K}_{1,2}^{k}
$$

These equations represent the WTT equations for weak MHD turbulence (with zero residual energy), which was first derived in Ref. [9] using the standard WTT technique.

Let us now turn back to the imaginary part of Eqs. (17). For validity of the WTT it is necessary that the frequency shift caused by nonlinear interactions is smaller than $\omega_{\mathbf{k}}$. Taking into account that, according to Eq. (18), $\eta_{\mathbf{k}} \sim k_{\perp}^{4} E^{-}\left(0, k_{\perp}\right) / \partial \omega_{\mathbf{k}} / \partial k_{z}$, an analysis similar to the one performed above shows that, in addition to Eq. (22), the following condition should be satisfied

$$
\frac{\eta_{\mathrm{k}}}{\omega_{\mathrm{k}}} \ll 1 .
$$

The validity conditions of the WTT in a form similar to Eqs. (22) and (26) were first derived in Ref. [19]. In general case, the validity criterium of the WTT was found to be 
identical to Eq. (26). The appearance of the additional condition (22) for the validity of the kinetic equation (24), is related to the degenerate character of the solution of the resonant condition $\omega_{\mathbf{k}}=\omega_{\mathbf{k}_{1}}-\omega_{\mathbf{k}_{2}}$, which implies $k_{2 z}=0$, and therefore requires the participation in nonlinear interactions of modes with very low frequencies [in the context of the performed analysis it is clear that $E^{-}\left(0, k_{\perp}\right)$ in Eq. (22) should be understood as the average energy density of the modes with $\left.k_{z} \lesssim \eta_{\mathbf{k}} /\left(\partial \omega_{\mathbf{k}} / \partial k_{z}\right)\right]$. If Eq. (26) holds but the intensity of the low frequency modes is very low, such that left hand side of Eq. (22) is much greater then unity, then the kinetic equation (24) is not valid. However, it can be shown that the WTT is still valid then and the nonlinear interactions are dominated by four wave interactions. The corresponding kinetic equation was derived in Ref. [5]. Introducing the characteristic velocity of the perturbations with a characteristic parallel length scale $l_{z} \sim 1 / k_{z}$ and perpendicular length scale $l_{\perp} \sim 1 / k_{\perp}$, respectively, as $v_{l}^{2} \sim k_{z} k_{\perp}^{2} E\left(k_{z}, k_{\perp}\right)$, the conditions (22) and (26) reduce to the following ones $k_{\perp} v_{l} / k_{z} V_{A} \ll 1$ and $\left[k_{\perp} v_{l} E\left(0, k_{\perp}\right)\right] /\left[k_{z} V_{A} E\left(k_{z}, k_{\perp}\right)\right] \ll 1$. Note that the first condition coincides with Eq. (3).

Another important temporal characteristic of the turbulence (together with $\tau_{a c}$ ) is the energy cascade timescale $\tau_{c a s}$ which represents the timescale of the energy cascade described by Eq. (24). A simple way of determining $\tau_{c a s}$ is the following (a mathematically more sound derivation can be found in Ref. [20]): in the case of stationary turbulence, the left hand side of Eq. (24) is zero. But the characteristic timescale of the energy cascade can be determined if we retain only the modes with $\left|\mathbf{k}_{1 \perp}\right|>\left|\mathbf{k}_{\perp}\right|$ on the right hand side of Eq. (24). The equation then obtained describes the energy transfer from the mode with a wave number $\mathbf{k}_{\perp}$ to the modes with higher perpendicular wave numbers. The right hand side can be estimated as $n^{+} / \tau_{\text {cas }}$. The analysis of the left hand side terms similar to the one performed for the estimation of $\eta_{\mathbf{k}}$ yields

$$
\tau_{c a s} \sim \tau_{a c},
$$

and consequently, the WTT always suggests $N \sim\left(\tau_{c a s} / \tau_{a c}\right)^{2} \sim 1$. These arguments show that the IK model, which implies $\tau_{a c} \sim\left(k V_{A}\right)^{-1}, \tau_{c a s} \sim V_{A} /\left(k v_{l}^{2}\right)$ and consequently $N \sim$ $\left(V_{A} / v_{l}\right)^{2} \gg 1$, are incompatible with the WTT. Note that in the considered isotropic case Eq. (3) leads to the (incorrect) conclusion that the turbulence is weak. On the other hand, because $\tau_{a c} k V_{A} \sim 1$ Eq. (26) yields that the IK model does not correspond to the weak turbulence limit.

As was shown above, the width of the resonant are is $\Delta k_{z} \sim\left(\eta_{\mathbf{k}} / \omega_{\mathbf{k}}\right) k_{z}$. Taking into 
account Eq. (26) this implies that physically nonlinear interactions in the framework of the WTT can be interpreted as resonant interactions among spatially very large wave packets [with characteristic size $\Delta l \sim\left(\omega_{\mathbf{k}} / \eta_{\mathbf{k}}\right) / k_{z}$ ]. Although the nonlinear interactions are weak, the interacting wave packets are very large such that an original wave packet decays before interacting wave packets pass through each other. Because the introduction of the Dirac delta function in Eq. (24) requires the limit $\eta_{\mathbf{k}} / \omega_{\mathbf{k}} \rightarrow 0$, it is sometimes stated that in the framework of the WTT the units of the nonlinear interactions are not wave packets of a finite spatial extent but spatially infinite Fourier harmonics [19].

\section{CONCLUSIONS}

Both strong and weak turbulence regimes of incompressible MHD turbulence were considered in the framework of the WCA. We showed that in the framework of the WTT the autocorrelation and cascade timescales are always of the same order of magnitude and, consequently, the WTT always suggests $N \gg 1$. Physically this is caused by the fact that the framework of the WTT can be interpreted as the resonant interaction among spatially very large wave packets [with characteristic size $\Delta l \sim\left(\omega_{\mathbf{k}} / \eta_{\mathbf{k}}\right) / k_{z}$ ]. Although the nonlinear interactions are weak, the interacting wave packets are very large such that an original wave packet decays before interacting wave packets pass through each other.

\section{Acknowledgments}

G.G. and S.M.M. acknowledge the hospitality of the Abdus Salam International Center for Theoretical Physics (ICTP) where part of the work was done. G.G. acknowledges partial support from INTAS grant 061000017-9258 and Georgian NSF grants ST06/4-096 and ST07/4-193. S.M.M.'s work was supported by USDOE Contract No. DE-FG0204ER-54742. These results were obtained in the framework of the projects GOA/2009009 (K.U.Leuven), G.0304.07 (FWO-Vlaanderen) and C 90205 (ESA Prodex 9). Financial support by the European Commission through the SOLAIRE Network (MTRN-CT-2006- 
035484) is gratefully acknowledged.

[1] P. S. Iroshnikov, Astron. Zh. 40, 742 (1963).

[2] R. H. Kraichnan, Phys. Fluids 8, 1385 (1965).

[3] J. V. Shebalin, W. H. Matthaeus and D. Montgomery, J. Plasma Phys. 29, 525 (1983).

[4] J. C. Higdon, Astrophys. J. 285, 109 (1984).

[5] S. Sridhar and P. Goldreich, Astrophys. J. 432, 612 (1994).

[6] P. Goldreich and S. Sridhar, Astrophys. J. 438, 763 (1995).

[7] C. S. Ng, and A. Bhattacharjee, Phys. Plasmas 4, 605 (1997).

[8] J. Cho and E. Vishniac, Astrophys. J. 539, 273 (2000).

[9] S. Galtier, S. V. Nazarenko, A. C. Newell, and A. Pouquet, J. Plasma Phys. 63, 447 (2000).

[10] J. Maron and P. Goldreich, Astrophys. J. 554, 1175 (2001).

[11] J. Cho, A. Lazarian and E. Vishniac, Astrophys. J. 564, 291 (2002).

[12] W.-C. Müller, D. Biskamp and R. Grappin, Phys. Rev. E 67, 066302 (2003).

[13] W.-C. Müller and R. Grappin, Phys. Rev. Lett. 95, 114502 (2005).

[14] S. Oughton and W. H. Matthaeus, Nonlin. Proc. in Geophysics 12, 299 (2005).

[15] A. Schekochihin, and S. C. Cowley, in Magnetohydrodynamics: Historical Evolution and trends, eds. S. Molokov et al., (Springer, 2005). astro-ph/0507686.

[16] A. Beresnyak and A. Lazarian, Astrophys. J. 640, L175 (2006).

[17] G. Gogoberidze, Phys. Plasmas 14, 022304 (2007).

[18] A. Beresnyak and A. Lazarian, Astrophys. J. 682, 1070 (2008).

[19] B. B. Kadomtsev, Plasma Turbulence (Academic, New York, 1965), p. 54.

[20] V. E. Zakharov, V. S. L'vov and G. Falkovich, Kolmogorov Spectra of Turbulence I (SpringerVerlag, Berlin 1992).

[21] D. C. Leslie, Developments in the Theory of Turbulence (Oxford University Press, 1973), p. 24, 93 .

[22] R. H. Kraichnan, J. Fluid Mech. 5, 497 (1959).

[23] J. A. Krommes, Phys. Reports 360, 1 (2002).

[24] Y. Kaneda, Phys. Fluids A 5, 2835 (1993). 